\title{
Scale dependence of alpha effect and turbulent diffusivity
}

\author{
A. Brandenburg ${ }^{1}$, K.-H. Rädler ${ }^{2}$, and M. Schrinner ${ }^{3}$ \\ 1 NORDITA, Roslagstullsbacken 23, 10691 Stockholm, Sweden \\ e-mail: brandenb@nordita.org \\ 2 Astrophysical Institute Potsdam, An der Sternwarte 16, 14482 Potsdam, Germany \\ 3 Max-Planck-Institut für Sonnensystemforschung, 37191 Katlenburg-Lindau, Germany
}

Received 8 January 2008 / Accepted 8 February 2008

\section{ABSTRACT}

\begin{abstract}
Aims. We determine the alpha effect and turbulent magnetic diffusivity for mean magnetic fields with profiles of different length scales from simulations of isotropic turbulence. We then relate these results to nonlocal formulations in which alpha and the turbulent magnetic diffusivity correspond to integral kernels.

Methods. We solve evolution equations for magnetic fields that give the response to imposed test fields. These test fields correspond to mean fields with various wavenumbers. Both an imposed fully helical steady flow consisting of a pattern of screw-like motions (Roberts flow) and time-dependent, statistically steady isotropic turbulence are considered. In the latter case the evolution equations are solved simultaneously with the momentum and continuity equations. The corresponding results for the electromotive force are used to calculate alpha and magnetic diffusivity tensors.

Results. For both, the Roberts flow under the second-order correlation approximation and the isotropic turbulence alpha and turbulent magnetic diffusivity are greatest on large scales and these values diminish toward smaller scales. In both cases, the alpha effect and turbulent diffusion kernels are approximated by exponentials, corresponding to Lorentzian profiles in Fourier space. For isotropic turbulence, the turbulent diffusion kernel is half as wide as the alpha effect kernel. For the Roberts flow beyond the second-order correlation approximation, the turbulent diffusion kernel becomes negative on large scales.
\end{abstract}

Key words. magnetohydrodynamics (MHD) - hydrodynamics - turbulence

\section{Introduction}

Stars and galaxies harbor magnetic fields whose scales are larger than those of the underlying turbulence. This phenomenon is successfully explained in terms of mean-field dynamo theory discussed in detail in a number of textbooks and reviews (e.g. Moffatt 1978; Krause \& Rädler 1980; Brandenburg \& Subramanian 2005a). In this context, velocity and magnetic fields are split into large-scale and small-scale components, $\boldsymbol{U}=\overline{\boldsymbol{U}}+\boldsymbol{u}$ and $\boldsymbol{B}=\overline{\boldsymbol{B}}+\boldsymbol{b}$, respectively. The crucial quantity of the theory is the mean electromotive force caused by smallscale fields, $\overline{\mathcal{E}}=\overline{\boldsymbol{u} \times \boldsymbol{b}}$. In many representations it is discussed under strongly simplifying assumptions. Often the relationship between the mean electromotive force and the mean magnetic field is tacitly taken both as (almost) local and as instantaneous; that is, in a given point in space and time, $\overline{\mathcal{E}}$ is considered as determined by $\overline{\boldsymbol{B}}$ and its first spatial derivatives in this point only. In addition, the possibility of a small-scale dynamo is ignored. Then the mean electromotive force is given by

$\overline{\mathcal{E}}_{i}=\alpha_{i j} \bar{B}_{j}+\eta_{i j k} \partial \bar{B}_{j} / \partial x_{k}$

with two tensors $\alpha_{i j}$ and $\eta_{i j k}$. If the turbulence is isotropic, the two tensors are isotropic, too; that is $\alpha_{i j}=\alpha \delta_{i j}$ and $\eta_{i j k}=\eta_{\mathrm{t}} \epsilon_{i j k}$ with two scalar coefficients $\alpha$ and $\eta_{\mathrm{t}}$. Then the expression (1) simplifies to

$\overline{\mathcal{E}}=\alpha \overline{\boldsymbol{B}}-\eta_{\mathrm{t}} \overline{\boldsymbol{J}}$,

where we denote $\boldsymbol{\nabla} \times \boldsymbol{B}$ simply by $\boldsymbol{J}$ (so that $\boldsymbol{J}$ is $\mu_{0}$ times the electric current density, where $\mu_{0}$ is the magnetic permeability of free space). The coefficient $\alpha$ is, unlike $\eta_{\mathrm{t}}$, only non-zero if the turbulence lacks mirror-symmetry. The coefficient $\eta_{\mathrm{t}}$ is referred to as the turbulent magnetic diffusivity.

In general, the mean electromotive force has the form

$\overline{\mathcal{E}}=\overline{\mathcal{E}}_{0}+\boldsymbol{K} \circ \overline{\boldsymbol{B}}$

where $\overline{\mathcal{E}}_{0}$ stands for a part of $\overline{\mathcal{E}}$ that is independent of $\overline{\boldsymbol{B}}$, and $\boldsymbol{K} \circ \overline{\boldsymbol{B}}$ denotes a convolution in space and time of a kernel $\boldsymbol{K}$ with $\overline{\boldsymbol{B}}$ (see, e.g., Krause \& Rädler 1980; Rädler 2000; Rädler \& Rheinhardt 2007). Due to this convolution, $\overline{\mathcal{E}}$ in a given point in space and time depends on $\overline{\boldsymbol{B}}$ in a certain neighborhood of this point, with the exception of future times. This corresponds to a modification of (1) such that also higher spatial and also time derivatives of $\overline{\boldsymbol{B}}$ occur.

In this paper we ignore the possibility of coherent effects resulting from small-scale dynamo action and therefore put $\overline{\boldsymbol{\varepsilon}}_{0}$ equal to zero. For the sake of simplicity we assume the connection between $\overline{\mathcal{E}}$ and $\overline{\boldsymbol{B}}$ to be instantaneous so that the convolution $\boldsymbol{K} \circ \overline{\boldsymbol{B}}$ only refers to space coordinates. The memory effect, which we thus ignore, has been studied previously by solving an evolution equation for $\overline{\mathcal{E}}$ (Blackman \& Field 2002).

For homogeneous isotropic turbulence, we may then write, analogously to (2),

$\overline{\mathcal{E}}=\hat{\alpha} \circ \overline{\boldsymbol{B}}-\hat{\eta}_{\mathrm{t}} \circ \overline{\boldsymbol{J}}$ 
or, in more explicit form,

$\overline{\mathcal{E}}(\boldsymbol{x})=\int\left[\hat{\alpha}(\xi) \overline{\boldsymbol{B}}(\boldsymbol{x}-\boldsymbol{\xi})-\hat{\eta}_{\mathrm{t}}(\xi) \overline{\boldsymbol{J}}(\boldsymbol{x}-\boldsymbol{\xi})\right] \mathrm{d}^{3} \xi$

with two functions $\hat{\alpha}$ and $\hat{\eta}_{\mathrm{t}}$ of $\xi=|\xi|$ that vanish for large $\xi$. The integration is in general over all $\xi$-space. Although $\overline{\mathcal{E}}$ and $\overline{\boldsymbol{B}}$, as well as $\hat{\alpha}$ and $\hat{\eta}_{\mathrm{t}}$, may depend on time, the argument $t$ is dropped everywhere. For a detailed justification of the relations (4) and (5), we refer to Appendix A. In the limit of a weak dependence of $\overline{\boldsymbol{B}}$ and $\overline{\boldsymbol{J}}$ on space coordinates, i.e. when the variations of $\overline{\boldsymbol{B}}(\boldsymbol{x}-\boldsymbol{\xi})$ and $\overline{\boldsymbol{J}}(\boldsymbol{x}-\boldsymbol{\xi})$ with $\boldsymbol{\xi}$ are small in the range of $\xi$ where $\hat{\alpha}(\xi)$ and $\hat{\eta}_{\mathrm{t}}(\xi)$ are markedly different from zero, the relations (4) or (5) turn into (2), and we see that $\alpha=\int \hat{\alpha}(\xi) \mathrm{d}^{3} \xi$ and $\eta_{\mathrm{t}}=\int \hat{\eta}_{\mathrm{t}}(\xi) \mathrm{d}^{3} \xi$.

At first glance, the representations (4) and (5) of $\overline{\mathcal{E}}$ look rather different from (3). Considering $\overline{\boldsymbol{J}}=\boldsymbol{\nabla} \times \overline{\boldsymbol{B}}$ and carrying out an integration by parts, we may however easily rewrite (5) into

$\overline{\mathcal{E}}_{i}(\boldsymbol{x})=\int K_{i j}(\boldsymbol{\xi}) \bar{B}_{j}(\boldsymbol{x}-\boldsymbol{\xi}) \mathrm{d}^{3} \xi$

with

$K_{i j}(\xi)=\hat{\alpha}(\xi) \delta_{i j}+\frac{1}{\xi} \frac{\partial \hat{\eta}_{\mathrm{t}}(\xi)}{\partial \xi} \epsilon_{i j k} \xi_{k}$.

We further note that due to the symmetry of $\hat{\alpha}(\xi)$ in $\xi$ only the part of $\overline{\boldsymbol{B}}(\boldsymbol{x}-\boldsymbol{\xi})$ that is symmetric in $\boldsymbol{\xi}$, i.e. the part that can be described by $\overline{\boldsymbol{B}}(\boldsymbol{x})$ and its derivatives of even order, contributes to the $\hat{\alpha}$ terms in (5) or in (6) and (7). The symmetry of $\hat{\eta}_{\mathrm{t}}(\xi)$ implies that only that part of $\overline{\boldsymbol{B}}(\boldsymbol{x}-\boldsymbol{\xi})$ that is antisymmetric in $\boldsymbol{\xi}$, which corresponds to the derivatives of $\overline{\boldsymbol{B}}(\boldsymbol{x})$ of odd order, contributes to the $\hat{\eta}_{\mathrm{t}}$ terms.

Finally, referring to a Cartesian coordinate system $(x, y, z)$, we define mean fields by averaging over all $x$ and $y$, so that in particular $\overline{\mathcal{E}}$ and $\overline{\boldsymbol{B}}$ only depend on $z$ and on time. Then (5) turns into

$\overline{\boldsymbol{E}}(z)=\int\left[\hat{\alpha}(\zeta) \overline{\boldsymbol{B}}(z-\zeta)-\hat{\eta}_{\mathrm{t}}(\zeta) \overline{\boldsymbol{J}}(z-\zeta)\right] \mathrm{d} \zeta$.

The functions $\hat{\alpha}(\zeta)$ and $\hat{\eta}_{\mathrm{t}}(\zeta)$ are just averages of $\hat{\alpha}(\xi)$ and $\hat{\eta}_{\mathrm{t}}(\xi)$ over all $\xi_{x}$ and $\xi_{y}$. They are therefore real and symmetric in $\xi_{z} \equiv$ $\zeta$. The integration in (8) is in general over all $\zeta$. The remark made in connection with (4) and (5) on the limit of weak dependencies of $\overline{\boldsymbol{B}}$ and $\overline{\boldsymbol{J}}$ on space coordinates applies analogously to (8). We now have $\alpha=\int \hat{\alpha}(\zeta) \mathrm{d} \zeta$ and $\eta_{\mathrm{t}}=\int \hat{\eta}_{\mathrm{t}}(\zeta) \mathrm{d} \zeta$.

Relation (8) can also be brought in a form analogous to (6) and (7),

$\bar{\varepsilon}_{i}(z)=\int K_{i j}(\zeta) \bar{B}_{j}(z-\zeta) \mathrm{d} \zeta$

with

$K_{i j}(\zeta)=\hat{\alpha}(\zeta) \delta_{i j}+\frac{\partial \hat{\eta}_{\mathrm{t}}(\zeta)}{\partial \zeta} \epsilon_{i j 3}$

The remarks made under (6) and (7) apply, now due to the symmetries of $\hat{\alpha}(\zeta)$ and $\hat{\eta}_{\mathrm{t}}(\zeta)$ in $\zeta$, analogously to (8)-(10).

It is useful to consider in addition to (8) the corresponding Fourier representation. We define the Fourier transformation in this paper by $Q(z)=\int \tilde{Q}(k) \exp (\mathrm{i} k z) \mathrm{d}(k / 2 \pi)$. Then this representation reads as

$\tilde{\overline{\mathcal{E}}}(k)=\tilde{\alpha}(k) \tilde{\overline{\boldsymbol{B}}}(k)-\tilde{\eta}_{\mathrm{t}}(k) \tilde{\overline{\boldsymbol{J}}}(k)$.
Both $\tilde{\alpha}(k)$ and $\tilde{\eta}_{\mathrm{t}}(k)$ are real quantities, and they are symmetric in $k$. The limit of weak dependencies of $\overline{\boldsymbol{B}}$ and $\overline{\boldsymbol{J}}$ on $z$ corresponds here to $k \rightarrow 0$, and we have $\alpha=\tilde{\alpha}(0)$ and $\eta_{\mathrm{t}}=\tilde{\eta}_{\mathrm{t}}(0)$. Detailed analytic expressions for $\hat{\alpha}(\zeta)$ and $\hat{\eta}_{\mathrm{t}}(\zeta)$, or $\tilde{\alpha}(k)$ and $\tilde{\eta}_{\mathrm{t}}(k)$, can be derived, e.g., from results presented in Krause \& Rädler (1980). A numerical determination of quantities corresponding to $\hat{\alpha}(\zeta)$ and $\hat{\eta}_{\mathrm{t}}(\zeta)$ has been attempted by Brandenburg $\&$ Sokoloff (2002) for shear flow turbulence.

In this paper, two specifications of the velocity field $\boldsymbol{u}$ will be considered. In the first case $\boldsymbol{u}$ is chosen such that it corresponds to a steady Roberts flow, which is periodic in $x$ and $y$ and independent of $z$. A mean-field theory of a magnetic field in fluid flows of this type, which are of course different from genuine turbulence, has been developed in the context of the Karlsruhe dynamo experiment (Rädler et al. 2002a,b; Rädler \& Brandenburg 2003). It turned out that the mean electromotive force $\overline{\mathcal{E}}$, except its $z$ component, satisfies relation (2) if any nonlocality in the above sense is ignored (see also Appendix B). Several analytical and numerical results are available for comparison with those of the present paper. In the second case $\boldsymbol{u}$ is understood as homogeneous, isotropic, statistically steady turbulence, for which the above explanations apply immediately. Employing the method developed by Schrinner et al. (2005, 2007), we will in both cases numerically calculate the functions $\tilde{\alpha}(k)$ and $\tilde{\eta}_{\mathrm{t}}(k)$, as well as $\hat{\alpha}(\zeta)$ and $\hat{\eta}_{\mathrm{t}}(\zeta)$

\section{The method}

We first relax the assumption of isotropic turbulence used in the Sect. 1 (but will later return to it). We remain, however, with the definition of mean fields by averaging over all $x$ and $y$. Then, as already roughly indicated above, $\bar{B}_{x}$ and $\bar{B}_{y}$ may only depend on $z$ and time but $\bar{B}_{z}$, because of $\boldsymbol{\nabla} \cdot \overline{\boldsymbol{B}}=0$, must be independent of $z$. Furthermore, all first-order spatial derivatives of $\overline{\boldsymbol{B}}$ can be expressed by the components of $\boldsymbol{\nabla} \times \overline{\boldsymbol{B}}$, that is, of $\overline{\boldsymbol{J}}$, where $\bar{J}_{z}=0$. Instead of (8) we then have

$\overline{\mathcal{E}}_{i}(z)=\int\left[\hat{\alpha}_{i j}(\zeta) \bar{B}_{j}(z-\zeta)-\hat{\eta}_{i j}(\zeta) \bar{J}_{j}(z-\zeta)\right] \mathrm{d} \zeta$,

and instead of (11),

$\tilde{\overline{\mathcal{E}}}_{i}(k)=\tilde{\alpha}_{i j}(k) \tilde{\bar{B}}_{j}(k)-\tilde{\eta}_{i j}(k) \tilde{\bar{J}}_{j}(k)$,

with real $\hat{\alpha}_{i j}(\zeta)$ and $\hat{\eta}_{i j}(\zeta)$, which are even in $\zeta$, and real $\tilde{\alpha}_{i j}(k)$ and $\tilde{\eta}_{i j}(k)$, which are even in $k$. A justification of these relations is given in Appendix A. We have further

$\tilde{\alpha}_{i j}(k)=\int \hat{\alpha}_{i j}(\zeta) \cos k \zeta \mathrm{d} \zeta, \quad \tilde{\eta}_{i j}(k)=\int \hat{\eta}_{i j}(\zeta) \cos k \zeta \mathrm{d} \zeta$.

Since $\bar{J}_{3}=0$, the $\hat{\eta}_{i 3}$, as well as the $\tilde{\eta}_{i 3}$, are of no interest.

In the following we restrict our attention to $\overline{\mathcal{E}}_{x}$ and $\overline{\mathcal{E}}_{y}$ and assume that $\bar{B}_{z}$ is equal to zero. We note that $\overline{\mathcal{E}}_{z}$ and the contributions of $\bar{B}_{z}$ to $\overline{\mathcal{E}}_{x}$ and $\overline{\mathcal{E}}_{y}$ are without interest for the mean-field induction equation, which only contains $\overline{\mathcal{E}}$ in the form $\boldsymbol{\nabla} \times \overline{\mathcal{E}}$; that is, they do not affect the evolution of $\overline{\boldsymbol{B}}$. We may formulate the above restriction in a slightly different way by saying that we consider in the following $\overline{\mathcal{E}}_{i}, \alpha_{i j}$, and $\eta_{i j}$, as well as $\tilde{\overline{\mathcal{E}}}_{i}, \tilde{\alpha}_{i j}$, and $\tilde{\eta}_{i j}$ only for $1 \leq i, j \leq 2$.

As for the mean-field treatment of a Roberts flow depending only on $x$ and $y$ (and not on $z$ ), we refer to the aforementioned studies (Rädler et al. 2002a,b; 
Rädler \& Brandenburg 2003). Following the ideas explained there, we may conclude that $\hat{\alpha}_{i j}(\zeta)=\hat{\alpha}(\zeta) \delta_{i j}$ and $\hat{\eta}_{i j}(\zeta)=\hat{\eta}_{\mathrm{t}}(\zeta) \delta_{i j}$ with functions $\hat{\alpha}$ and $\hat{\eta}_{\mathrm{t}}$ of $\zeta$, and analogously $\tilde{\alpha}_{i j}(k)=\tilde{\alpha}(k) \delta_{i j}$ and $\tilde{\eta}_{i j}(k)=\tilde{\eta}_{\mathrm{t}}(k) \delta_{i j}$ with functions $\tilde{\alpha}$ and $\tilde{\eta}_{\mathrm{t}}$ of $k$, all for $1 \leq i, j \leq 2$. For obvious reasons the same is true for homogeneous isotropic turbulence.

\subsection{Test-field method}

We calculate the $\tilde{\alpha}_{i j}(k)$ and $\tilde{\eta}_{i j}(k)$, or $\tilde{\alpha}(k)$ and $\tilde{\eta}(k)$, numerically by employing the test-field method of Schrinner et al. (2005, 2007). It was originally developed to calculate the full $\alpha$ and $\eta$ tensors (in the sense of (1)) for convection in a spherical shell. Brandenburg (2005) employed this method to obtain results for stratified shear flow turbulence in a local cartesian domain using the shearing sheet approximation. More recently, Sur et al. (2008) calculated the dependencies of $\alpha$ and $\eta_{\mathrm{t}}$ in this way for isotropic turbulence on the magnetic Reynolds number, and Brandenburg et al. (2008) calculated the magnetic diffusivity tensor for rotating and shear flow turbulence. However, in all these cases no nonlocality in the connection between $\overline{\boldsymbol{E}}$ and $\overline{\boldsymbol{B}}$ has been taken into account.

Following the idea of Schrinner et al., we first derive expressions for $\overline{\mathcal{E}}$ with several specific $\overline{\boldsymbol{B}}$, which we call "test fields". We denote the latter by $\overline{\boldsymbol{B}}^{p q}$ and define ${ }^{1}$

$\overline{\boldsymbol{B}}^{1 \mathrm{c}}=B(\cos k z, 0,0), \quad \overline{\boldsymbol{B}}^{2 \mathrm{c}}=B(0, \cos k z, 0)$,

$\overline{\boldsymbol{B}}^{1 \mathrm{~s}}=B(\sin k z, 0,0), \quad \overline{\boldsymbol{B}}^{2 \mathrm{~s}}=B(0, \sin k z, 0)$,

with any constant $B$ and any fixed value of $k$. We then replace $\overline{\boldsymbol{B}}$ and $\overline{\boldsymbol{J}}$ in (12) by $\overline{\boldsymbol{B}}^{p \mathrm{c}}$ and $\boldsymbol{\nabla} \times \overline{\boldsymbol{B}}^{p \mathrm{c}}$ or by $\overline{\boldsymbol{B}}^{p \mathrm{~s}}$ and $\boldsymbol{\nabla} \times \overline{\boldsymbol{B}}^{p \mathrm{~s}}$. Denoting the corresponding $\overline{\mathcal{E}}$ by $\overline{\mathcal{E}}^{p c}$ or by $\overline{\mathcal{E}}^{p s}$, respectively, and using (14) we find

$\overline{\mathcal{E}}_{i}^{p \mathrm{c}}(z)=B\left[\tilde{\alpha}_{i p}(k) \cos k z-\tilde{\eta}_{i p}^{\dagger}(k) k \sin k z\right]$,

$\bar{\varepsilon}_{i}^{p s}(z)=B\left[\tilde{\alpha}_{i p}(k) \sin k z+\tilde{\eta}_{i p}^{\dagger}(k) k \cos k z\right]$,

for $1 \leq i, p \leq 2$, where

$\tilde{\eta}_{i p}^{\dagger}=\tilde{\eta}_{i l} \epsilon_{l p 3}=\left(\begin{array}{ll}-\tilde{\eta}_{12} & \tilde{\eta}_{11} \\ -\tilde{\eta}_{22} & \tilde{\eta}_{21}\end{array}\right)$.

From this we conclude that

$\tilde{\alpha}_{i j}(k)=B^{-1}\left[\overline{\mathcal{E}}_{i}^{j \mathrm{c}}(z) \cos k z+\overline{\mathcal{E}}_{i}^{j \mathrm{~s}}(z) \sin k z\right]$,

$\tilde{\eta}_{i j}^{\dagger}(k)=-(k B)^{-1}\left[\overline{\mathcal{E}}_{i}^{j \mathrm{c}}(z) \sin k z-\overline{\mathcal{E}}_{i}^{j \mathrm{~s}}(z) \cos k z\right]$,

for $1 \leq i, j \leq 2$.

These relations allow us to calculate the $\tilde{\alpha}_{i j}$ and $\tilde{\eta}_{i j}$ if the $\overline{\mathcal{E}}_{i}^{p q}$ with $1 \leq i, p \leq 2$ for both $q=\mathrm{c}$ and $q=\mathrm{s}$ are known. In preparing the numerical calculation, we start from the induction equation. Its uncurled form reads

$\frac{\partial \boldsymbol{A}}{\partial t}=\boldsymbol{U} \times \boldsymbol{B}-\eta \boldsymbol{J}$

where $\boldsymbol{A}$ is the magnetic vector potential, $\boldsymbol{B}=\boldsymbol{\nabla} \times \boldsymbol{A}$, and $\boldsymbol{J}=$ $\boldsymbol{\nabla} \times \boldsymbol{B}$. Here the Weyl gauge of $\boldsymbol{A}$ is used; that is, an additional

1 The notation used here differs slightly from that in Brandenburg et al. (2008), where test fields $\overline{\boldsymbol{B}}^{p}$ were originally introduced, and only later were the two versions $\overline{\boldsymbol{B}}^{p \mathrm{c}}$ and $\overline{\boldsymbol{B}}^{p \mathrm{~s}}$ considered. gradient in (19) has been ignored. Taking the average of (19) we obtain

$\frac{\partial \overline{\boldsymbol{A}}}{\partial t}=\overline{\boldsymbol{U}} \times \overline{\boldsymbol{B}}+\overline{\boldsymbol{u} \times \boldsymbol{b}}-\eta \overline{\boldsymbol{J}}$

From (19) and (20) we conclude

$\frac{\partial \boldsymbol{a}}{\partial t}=\overline{\boldsymbol{U}} \times \boldsymbol{b}+\boldsymbol{u} \times \overline{\boldsymbol{B}}+\boldsymbol{u} \times \boldsymbol{b}-\overline{\boldsymbol{u} \times \boldsymbol{b}}-\eta \boldsymbol{j}$,

where $\boldsymbol{a}=\boldsymbol{A}-\overline{\boldsymbol{A}}$ and $\boldsymbol{j}=\boldsymbol{J}-\overline{\boldsymbol{J}}=\boldsymbol{\nabla} \times \boldsymbol{b}$.

For calculating the $\overline{\mathcal{E}}^{p q}$ we are interested in the $\boldsymbol{b}^{p q}=\boldsymbol{\nabla} \times \boldsymbol{a}^{p q}$, which occur in response to the test fields $\overline{\boldsymbol{B}}^{p q}$. Specifying (21) in that sense we obtain ${ }^{2}$

$\frac{\partial \boldsymbol{a}^{p q}}{\partial t}=\overline{\boldsymbol{U}} \times \boldsymbol{b}^{p q}+\boldsymbol{u} \times \overline{\boldsymbol{B}}^{p q}+\boldsymbol{u} \times \boldsymbol{b}^{p q}-\overline{\boldsymbol{u} \times \boldsymbol{b}^{p q}}-\eta \boldsymbol{j}^{p q}$.

In the following, equations of this type are referred to as "testfield equations".

So far, no approximation has been made such as the second-order correlation approximation (SOCA), also known as first order smoothing approximation. If we were to make this assumption, terms that are nonlinear in the fluctuations would be neglected and (22) would simplify to

$\frac{\partial \boldsymbol{a}^{p q}}{\partial t}=\overline{\boldsymbol{U}} \times \boldsymbol{b}^{p q}+\boldsymbol{u} \times \overline{\boldsymbol{B}}^{p q}-\eta \boldsymbol{j}^{p q} \quad$ (for SOCA only)

In the following SOCA results will be shown in some examples for comparison only.

In the general case, as well as under SOCA, the $\tilde{\alpha}_{i j}$ and $\tilde{\eta}_{i j}$ are to be calculated from $\overline{\boldsymbol{\varepsilon}}^{p q}=\overline{\boldsymbol{u} \times \boldsymbol{b}^{p q}}$. More details of the numerical calculations of the $\overline{\mathcal{E}}^{p q}$ will be given below in Sect. 2.3.

Returning once more to (18), we note that the $\overline{\mathcal{E}}^{p q}$ depend on both $k$ and $z$ introduced with the $\overline{\boldsymbol{B}}^{p q}$. As a consequence of fluctuating averages, they may also depend on time $t$. The $\tilde{\alpha}_{i j}$ and $\tilde{\eta}_{i j}$ however should depend on $k$ but no longer on $z$ and $t$. We remove the latter dependencies of our results by averaging $\tilde{\alpha}_{i j}$ and $\tilde{\eta}_{i j}$ over $z$ and $t$. For the Roberts flow there should be no such $z$ or $t$ dependencies.

The relations (18) allow the determination of all components of $\tilde{\alpha}_{i j}$ and $\tilde{\eta}_{i j}$ with $1 \leq i, j \leq 2$. We know already that $\tilde{\alpha}_{i j}=\tilde{\alpha} \delta_{i j}$ and $\tilde{\eta}_{i j}=\tilde{\eta}_{\mathrm{t}} \delta_{i j}$, that is, $\tilde{\alpha}_{11}=\tilde{\alpha}_{22}=\tilde{\alpha}, \tilde{\eta}_{11}=\tilde{\eta}_{22}=\tilde{\eta}$ and $\tilde{\alpha}_{12}=\tilde{\alpha}_{21}=\tilde{\eta}_{12}=\tilde{\eta}_{21}=0$. We may therefore determine $\tilde{\alpha}$ and $\tilde{\eta}_{\mathrm{t}}$ according to $\tilde{\alpha}=\tilde{\alpha}_{11}$ and $\tilde{\eta}_{\mathrm{t}}=\tilde{\eta}_{11}$ by using the two test fields $\overline{\boldsymbol{B}}^{1 q}$ and the relations (18) with $i=j=1$ only.

\subsection{Flow fields}

\subsubsection{Roberts flow}

We consider here a special form of a steady flow that, in view of its dynamo action, has already been studied by Roberts (1972). It has no mean part, $\overline{\boldsymbol{U}}=\mathbf{0}$, and $\boldsymbol{u}$ is given by

$\boldsymbol{u}=-\hat{z} \times \nabla \psi+k_{\mathrm{f}} \psi \hat{z}$

where

$\psi=\left(u_{0} / k_{0}\right) \cos k_{0} x \cos k_{0} y, \quad k_{\mathrm{f}}=\sqrt{2} k_{0}$

${ }^{2}$ The $\overline{\boldsymbol{U}}$ term is incorrect in the corresponding expression (27) of Brandenburg (2005). This did not affect his results, because $\overline{\boldsymbol{U}}=0$. 
with some constant $k_{0}$. The vorticity of the flow is $\boldsymbol{\nabla} \times \boldsymbol{u}=k_{\mathrm{f}} \boldsymbol{u}$, so the helicity is maximal; this implies $\boldsymbol{u} \cdot \boldsymbol{\nabla} \times \boldsymbol{u}=k_{\mathrm{f}} \boldsymbol{u}^{2}$. The component form of $\boldsymbol{u}$ as defined by (24) and (25) reads

$\boldsymbol{u}=u_{0}\left(-\cos k_{0} x \sin k_{0} y, \sin k_{0} x \cos k_{0} y, \sqrt{2} \cos k_{0} x \cos k_{0} y\right)$.

We note that $\overline{\boldsymbol{u}^{2}}=u_{0}^{2}$.

\subsubsection{Turbulence}

Next, we consider isotropic, weakly compressible turbulence and use an isothermal equation of state with constant speed of sound, $c_{\mathrm{s}}$. Considering first the full velocity field $\boldsymbol{U}=\overline{\boldsymbol{U}}+\boldsymbol{u}$, we thus accept the momentum equation in the form

$\frac{\partial \boldsymbol{U}}{\partial t}=-\boldsymbol{U} \cdot \boldsymbol{\nabla} \boldsymbol{U}-c_{\mathrm{s}}^{2} \boldsymbol{\nabla} \ln \rho+\boldsymbol{f}+\rho^{-1} \boldsymbol{\nabla} \cdot 2 \rho v \boldsymbol{S}$,

where $f$ is a random forcing function consisting of circularly polarized plane waves with positive helicity and random direction, and $\mathrm{S}_{i j}=\frac{1}{2}\left(U_{i, j}+U_{j, i}\right)-\frac{1}{3} \delta_{i j} \boldsymbol{\nabla} \cdot \boldsymbol{U}$ is the traceless rate of strain tensor. The forcing function is chosen such that the magnitudes of the wavevectors, $\left|\boldsymbol{k}_{\mathrm{f}}\right|$, are in a narrow interval around an average value, which is again denoted simply by $k_{\mathrm{f}}$. The corresponding scale, $k_{\mathrm{f}}^{-1}$, is also referred to as the outer scale or the energy-carrying scale of the turbulence. More details concerning the forcing function are given in the appendix of Brandenburg \& Subramanian (2005b). With the intention of studying the mean electromotive force in the purely kinematic limit, the Lorentz force has been ignored.

In addition to the momentum equation we use the continuity equation in the form

$\frac{\partial \ln \rho}{\partial t}=-\boldsymbol{U} \cdot \boldsymbol{\nabla} \ln \rho-\boldsymbol{\nabla} \cdot \boldsymbol{U}$

In all simulations presented in this paper the strength of the forcing is adjusted such that the flow remains clearly subsonic, that is, the mean Mach number remains below 0.2. Hence for all practical purposes the flow can be considered incompressible (Dobler et al. 2003). In these simulations no mean flow develops, that is $\overline{\boldsymbol{U}}=\mathbf{0}$, so $\boldsymbol{U}=\boldsymbol{u}$.

\subsection{Simulations}

The relevant equations are solved in a computational domain of size $L \times L \times L$ using periodic boundary conditions. In the case of the Roberts flow (26), we fix $L$ by $L=2 \pi / k_{0}$. The test-field Eqs. (22) with $p=1, q=\mathrm{c}$, and $q=\mathrm{s}$ (six equations altogether) are solved numerically. With turbulence in the kinematic regime, the four Eqs. (27) and (28) for $\boldsymbol{U}$ and $\ln \rho$ are solved, together with these six test-field Eqs. (22).

Due to the finiteness of the domain in $z$ direction and the periodic boundary conditions, quantities like $\overline{\mathcal{E}}$ and $\overline{\boldsymbol{B}}$ have to be considered as functions that are periodic in $z$. The Fourier integrals used for representing these quantities, $Q(z)=\int \tilde{Q}(k) \exp (\mathrm{i} k z) \mathrm{d}(k / 2 \pi)$, turn into Fourier series, $Q(z)=$ $\sum Q_{n} \exp \left(\mathrm{i} k_{n} z\right) / L$, where $k_{n}=2 \pi n / L$ and the summation is over $n=0, \pm 1, \pm 2, \ldots$ For this reason only discrete values of $k$, i.e. $k=k_{n}$, are admissible in (13)-(18). In this framework we may determine the $\tilde{\alpha}$ and $\tilde{\eta}_{\mathrm{t}}$ only for these $k_{n}$.

As explained above, the test-field procedure yields $\tilde{\alpha}$ and $\tilde{\eta}_{\mathrm{t}}$ not as functions of $k$ alone. They may also show some dependence on $z$ and $t$. After having averaged over $z$, time averages are then taken over a suitable stretch of the full time series where these averages are approximately steady. We use the
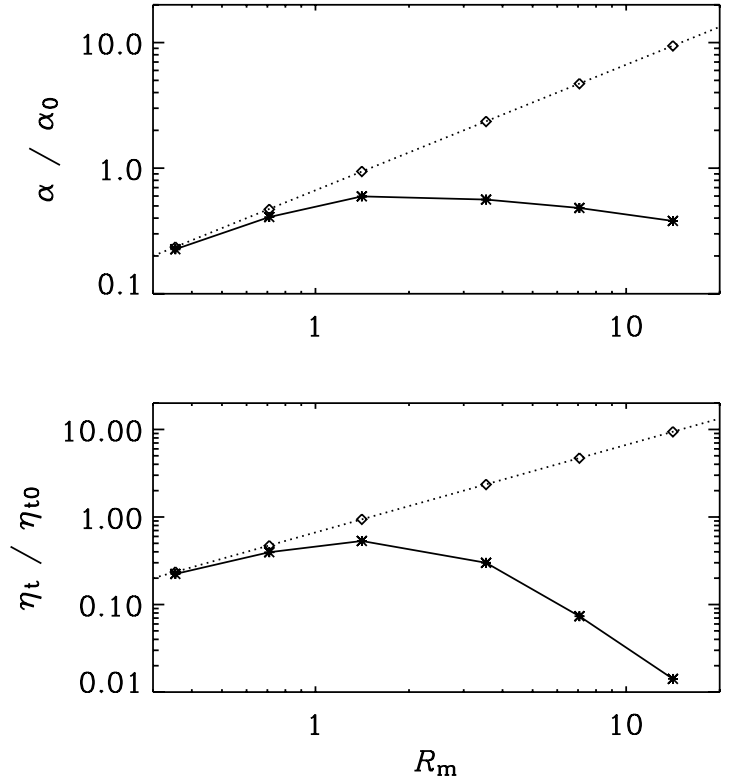

Fig. 1. Dependencies of the normalized $\alpha$ and $\eta_{\mathrm{t}}$ on $R_{\mathrm{m}}$ for the Roberts flow in the general case, i.e. independent of SOCA (solid lines), and in SOCA (dotted lines).

time series also to calculate error bars as the maximum departure between these averages and the averages obtained from one of three equally long subsections of the full time series.

In all cases the simulations were carried out using the PENCIL $\mathrm{CODE}^{3}$, which is a high-order finite-difference code (sixth order in space and third order in time) for solving the compressible hydromagnetic equations, together with the test-field equations. In the case of the Roberts flow, of course, only the test-field equations are being solved.

\section{Results}

\subsection{Roberts flow}

Let us first recall some findings of earlier works, such as Rädler (2002a,b). We use here the definitions

$\alpha_{0}=-\frac{1}{2} u_{0}, \quad \eta_{\mathrm{t} 0}=\frac{1}{2} u_{0} / k_{\mathrm{f}}, \quad R_{\mathrm{m}}=u_{0} / \eta k_{\mathrm{f}}$.

Adapting the results of analytic calculations in the framework of SOCA to the assumptions and notations of the present paper (see Appendix B), we have

$\alpha / \alpha_{0}=\eta_{\mathrm{t}} / \eta_{\mathrm{t} 0}=R_{\mathrm{m}}$.

Moreover, in the general case, without restriction to SOCA, it was found that

$\alpha=\alpha_{0} R_{\mathrm{m}} \phi\left(\sqrt{2} R_{\mathrm{m}}\right)$

with a function $\phi$ satisfying $\phi(0)=1$ and vanishing with growing argument. This function has been calculated numerically and is plotted, e.g., in Rädler et al. (2002a,b).

Figure 1 shows results for $\alpha$ and $\eta_{\mathrm{t}}$ obtained both by general test-field calculations using (22) and under the restriction to SOCA using (23). These results for $\alpha$ agree completely with both (30) and (31), and those for $\eta_{\mathrm{t}}$ agree completely with (30). Unfortunately we have no analytical results for $\eta_{\mathrm{t}}$ beyond SOCA.

${ }^{3}$ http://www.nordita.org/software/pencil-code 

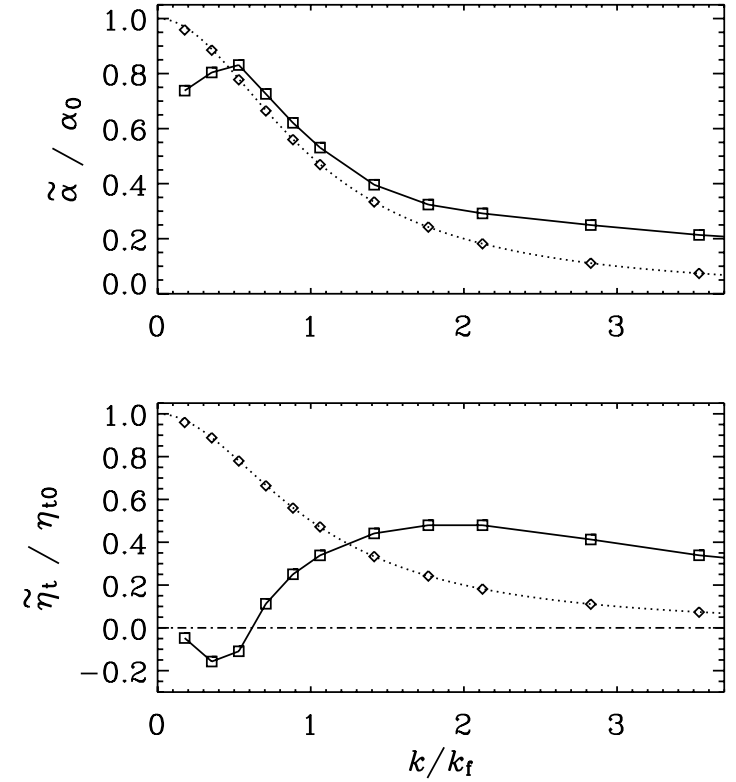

Fig. 2. Dependences of the normalized $\tilde{\alpha}$ and $\tilde{\eta}_{\mathrm{t}}$ on $k / k_{\mathrm{f}}$ for the Roberts flow with $R_{\mathrm{m}}=10 / \sqrt{2} \approx 7.1$ (solid lines), compared with normalized SOCA results for $\tilde{\alpha} / R_{\mathrm{m}}$ and $\tilde{\eta}_{\mathrm{t}} / R_{\mathrm{m}}$, which are independent of $R_{\mathrm{m}}$ (dotted lines).

Proceeding now to $\tilde{\alpha}(k)$ and $\tilde{\eta}_{\mathrm{t}}(k)$, we first note that in SOCA, as shown in Appendix C,

$\tilde{\alpha}(k)=\frac{\alpha_{0} R_{\mathrm{m}}}{1+\left(k / k_{\mathrm{f}}\right)^{2}}, \quad \tilde{\eta}_{\mathrm{t}}(k)=\frac{\eta_{\mathrm{t} 0} R_{\mathrm{m}}}{1+\left(k / k_{\mathrm{f}}\right)^{2}}$.

The corresponding $\hat{\alpha}(\zeta)$ and $\hat{\eta}_{\mathrm{t}}(\zeta)$, again in SOCA, read as

$\hat{\alpha}(\zeta)=\frac{1}{2} \alpha_{0} k_{\mathrm{f}} R_{\mathrm{m}} \exp \left(-k_{\mathrm{f}}|\zeta|\right), \quad \hat{\eta}_{\mathrm{t}}(\zeta)=\frac{1}{2} \eta_{\mathrm{t} 0} k_{\mathrm{f}} R_{\mathrm{m}} \exp \left(-k_{\mathrm{f}}|\zeta|\right)$.

In Fig. 2 results of test-field calculations for the functions $\tilde{\alpha}(k)$ and $\tilde{\eta}_{\mathrm{t}}(k)$ with $R_{\mathrm{m}}=10 / \sqrt{2} \approx 7.1$ are shown. We note that $\tilde{\eta}_{\mathrm{t}}$ becomes negative for small $k$. The same has been observed with another similar flow of the Roberts type (Rädler \& Brandenburg 2003). In the present case we have $\eta / \eta_{t 0}=2 / R_{\mathrm{m}} \approx 0.28$, which is larger than the most negative value of $\tilde{\eta}_{\mathrm{t}} / \eta_{\mathrm{t} 0}$. Therefore the total magnetic diffusivity is still positive. For comparison, SOCA results obtained in two different ways are also shown: those according to the analytic relations (32) and those calculated numerically by the test-field method with (23). Both agree very well with each other.

To obtain the results for the kernels $\hat{\alpha}(\zeta)$ and $\hat{\eta}_{\mathrm{t}}(\zeta)$, we have numerically calculated integrals as in (14) using the data plotted in Fig. 2. The results are represented in Fig. 3. Again, analytical and numerical SOCA results are shown for comparison. Note that the profiles of $\hat{\alpha}(\zeta)$ and $\hat{\eta}_{\mathrm{t}}(\zeta)$ beyond SOCA are rather narrow compared with those under SOCA, and that of $\hat{\eta}_{\mathrm{t}}(\zeta)$ even narrower than that of $\hat{\alpha}(\zeta)$.

\subsection{Isotropic turbulence}

Results for homogeneous isotropic turbulence were obtained by solving the hydrodynamic Eqs. (27) and (28) simultaneously with the test-field Eq. (22) in a domain of size $L \times L \times L$. The forcing wavenumbers $k_{\mathrm{f}}$ are fixed by $k_{\mathrm{f}} / k_{1}=5$ and 10 . Instead of the definitions (29), we now use

$\alpha_{0}=-\frac{1}{3} u_{\mathrm{rms}}, \quad \eta_{\mathrm{t} 0}=\frac{1}{3} u_{\mathrm{rms}} / k_{\mathrm{f}}, \quad R_{\mathrm{m}}=u_{\mathrm{rms}} / \eta k_{\mathrm{f}}$.
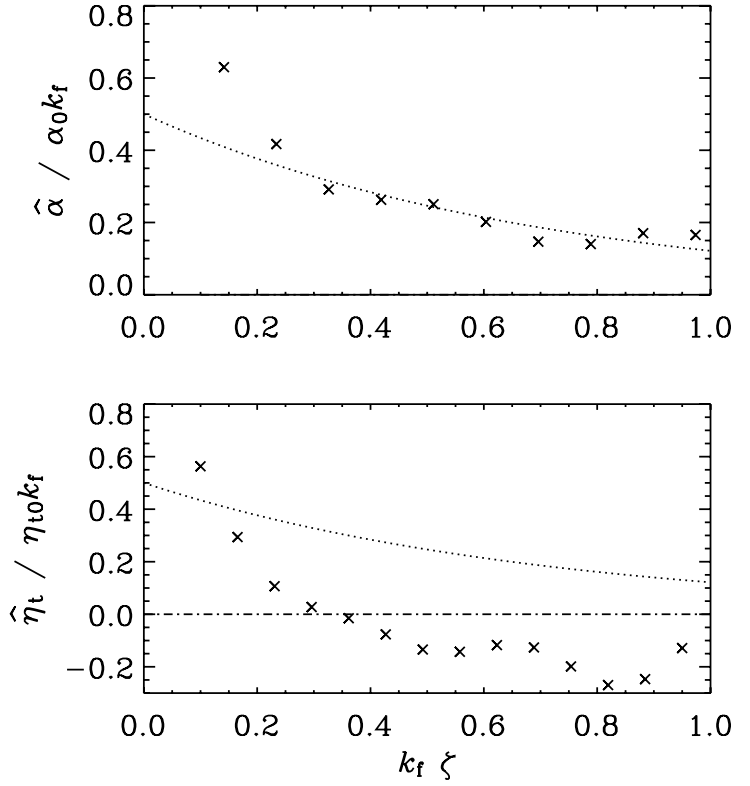

Fig. 3. Normalized integral kernels $\hat{\alpha}$ and $\hat{\eta}_{\mathrm{t}}$ versus $k_{\mathrm{f}} \zeta$ for the Roberts flow with $R_{\mathrm{m}}=10 / \sqrt{2} \approx 7.1$ (crosses), compared with normalized SOCA results for $\hat{\alpha} / R_{\mathrm{m}}$ and $\hat{\eta}_{\mathrm{t}} / R_{\mathrm{m}}$, which are independent of $R_{\mathrm{m}}$ (dotted lines).

Within this framework the dependence of $\alpha$ and $\eta_{\mathrm{t}}$ on $R_{\mathrm{m}}$ has been studied by Sur et al. (2008). They consider two cases, one with $v / \eta=0.1$ and another with $u_{\mathrm{rms}} / v k_{\mathrm{f}}=2.2$. Remarkably, they find that $\alpha / \alpha_{0}$ and $\eta / \eta_{0}$ approach unity for $R_{\mathrm{m}} \gg 1$.

Figure 4 shows results for $\tilde{\alpha}(k)$ and $\tilde{\eta}_{\mathrm{t}}(k)$ with $v / \eta=1$. Both $\tilde{\alpha}$ and $\tilde{\eta}_{\mathrm{t}}$ decrease monotonously with increasing $|k|$. The two values of $\tilde{\alpha}$ for a given $k / k_{\mathrm{f}}$ but different $k_{\mathrm{f}} / k_{1}$ and $R_{\mathrm{m}}$ are always very close together. The functions $\tilde{\alpha}(k)$ and $\tilde{\eta}_{\mathrm{t}}(k)$ are well represented by Lorentzian fits of the form

$\tilde{\alpha}(k)=\frac{\alpha_{0}}{1+\left(k / k_{\mathrm{f}}\right)^{2}}, \quad \tilde{\eta}_{\mathrm{t}}(k)=\frac{\eta_{\mathrm{t} 0}}{1+\left(k / 2 k_{\mathrm{f}}\right)^{2}}$.

Note the different dependencies of $\tilde{\alpha}$ and $\tilde{\eta}_{\mathrm{t}}$ on $k / k_{\mathrm{f}}$.

In Fig. 5 the kernels $\hat{\alpha}(\zeta)$ and $\hat{\eta}_{\mathrm{t}}(\zeta)$ are depicted, again with $v / \eta=1$, obtained by calculating numerically integrals as in (14). Also shown are the Fourier transforms of the Lorentzian fits,

$\hat{\alpha}(\zeta)=\frac{1}{2} \alpha_{0} k_{\mathrm{f}} \exp \left(-k_{\mathrm{f}}|\zeta|\right), \quad \hat{\eta}_{\mathrm{t}}(\zeta)=\eta_{\mathrm{t} 0} k_{\mathrm{f}} \exp \left(-2 k_{\mathrm{f}}|\zeta|\right)$.

Evidently, the profile of $\hat{\eta}_{\mathrm{t}}$ is half as wide as that of $\hat{\alpha}$. This corresponds qualitatively to our observation with the Roberts flow beyond SOCA, see the crosses in Fig. 3. There is, however, no counterpart to the negative values of $\hat{\eta}_{\mathrm{t}}$ that occur in the example of the Roberts flow.

The results presented in Figs. 4 and 5 show no noticeable dependencies on $R_{\mathrm{m}}$. Although we have not performed any systematic survey in $R_{\mathrm{m}}$, we interpret this as an extension of the abovementioned results of Sur et al. (2008) for $\alpha$ and $\eta_{\mathrm{t}}$ to the integral kernels $\hat{\alpha}$ and $\hat{\eta}_{\mathrm{t}}$. Of course, this should also be checked with higher values of $R_{\mathrm{m}}$. Particularly interesting would be a confirmation of different widths for the profiles of $\hat{\alpha}$ and $\hat{\eta}_{\mathrm{t}}$.

\section{Discussion}

Our results are important for calculating mean-field dynamo models. The mean-field induction equation governing $\overline{\boldsymbol{B}}$, here 

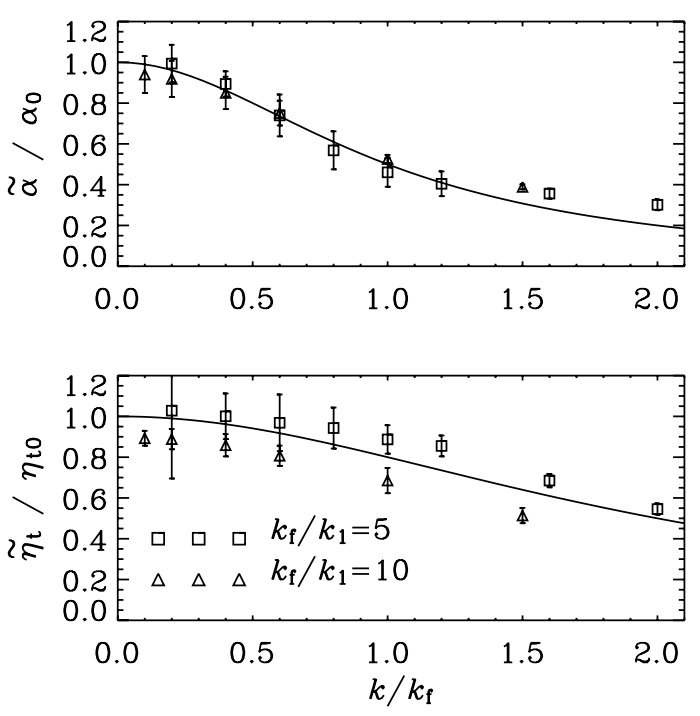

Fig. 4. Dependences of the normalized $\tilde{\alpha}$ and $\tilde{\eta}_{\mathrm{t}}$ on the normalized wavenumber $k / k_{\mathrm{f}}$ for isotropic turbulence forced at wavenumbers $k_{\mathrm{f}} / k_{1}=5$ with $R_{\mathrm{m}}=10$ (squares) and $k_{\mathrm{f}} / k_{1}=10$ with $R_{\mathrm{m}}=3.5$ (triangles), all with $v / \eta=1$. The solid lines give the Lorentzian fits (35).

defined as an average over $x$ and $y$, with $\overline{\mathcal{E}}$ according to (8), allows solutions of the form $\operatorname{Re}\left[\overline{\boldsymbol{B}}_{0} \exp (\mathrm{i} k z+\lambda t)\right], \bar{B}_{0 z}=0$, with the growth rate

$\lambda=-\left[\eta+\tilde{\eta}_{\mathrm{t}}(k)\right] k^{2} \pm \tilde{\alpha}(k) k$.

A dynamo occurs if $\lambda$ is non-negative. Since $\tilde{\alpha} \leq 0$ in all examples considered this occurs with the lower sign, and we focus attention on this case only. In the limit of a local connection between $\overline{\mathcal{E}}$ and $\overline{\boldsymbol{B}}$, the $\tilde{\eta}_{\mathrm{t}}(k)$ and $\tilde{\alpha}(k)$ turn into $\tilde{\eta}_{\mathrm{t}}(0)$ and $\tilde{\alpha}(0)$, respectively.

When using the definitions (29) for the Roberts flow or (34) for isotropic turbulence, we may write (37) in the form

$\lambda=\eta_{\mathrm{t} 0} k_{\mathrm{f}}^{2}\left\{-\left[\frac{\gamma}{R_{\mathrm{m}}}+\frac{\tilde{\eta}_{\mathrm{t}}\left(k / k_{\mathrm{f}}\right)}{\eta_{\mathrm{t} 0}}\right] \frac{k}{k_{\mathrm{f}}}+\frac{\tilde{\alpha}\left(k / k_{\mathrm{f}}\right)}{\alpha_{0}}\right\} \frac{k}{k_{\mathrm{f}}}$,

where $\gamma=2$ for the Roberts case and $\gamma=3$ for the isotropic case. Since $\tilde{\eta}_{\mathrm{t}}$ and $\tilde{\alpha}$ only depend on $k$ via $k / k_{\mathrm{f}}$ we have chosen the arguments $k / k_{\mathrm{f}}$.

Consider first the Roberts flow, that is, (38) with $\gamma=2$. Clearly $\lambda$ is non-negative in some interval $0 \leq k / k_{\mathrm{f}} \leq \kappa_{0}$ and it takes a maximum there. Dynamos with $k / k_{\mathrm{f}}>\kappa_{0}$ are impossible. Of course, $\kappa_{0}$ depends on $R_{\mathrm{m}}$. With the analytic SOCA results (32), we find $\kappa_{0}=\frac{1}{2} R_{\mathrm{m}}^{2}$ for small $R_{\mathrm{m}}$ and that $\kappa_{0}$ grows monotonically with $R_{\mathrm{m}}$, approaching unity in the limit of large $R_{\mathrm{m}}$. For small $R_{\mathrm{m}}$, a dynamo can work only with small $k / k_{\mathrm{f}}$, that is, with scales of the mean magnetic field that are much larger than the size of a flow cell. Furthermore, $\kappa_{0}$ never exceeds the corresponding values for vanishing nonlocal effect, which is $\frac{1}{2} R_{\mathrm{m}}^{2} /\left(1+\frac{1}{2} R_{\mathrm{m}}^{2}\right)$. In that sense the nonlocal effect favors smaller $k$, that is, larger scales of the mean magnetic field. With the numerical results beyond SOCA represented in Fig. 2, with $R_{\mathrm{m}}=10 / \sqrt{2}$, we have $\kappa_{0} \approx 0.90 \ldots 0.95$, again a value less than unity. In this case, too, a dynamo does not work with scales of the mean magnetic field smaller than that of a flow cell. There is no crucial impact of the negative values of $\tilde{\eta}_{\mathrm{t}}$ for $k / k_{\mathrm{f}}<0.8$ on the dynamo.

We proceed now to isotropic turbulence and consider (38) with $\gamma=3$. Again, $\lambda$ is non-zero in an interval $0 \leq k / k_{\mathrm{f}} \leq \kappa_{0}$ and
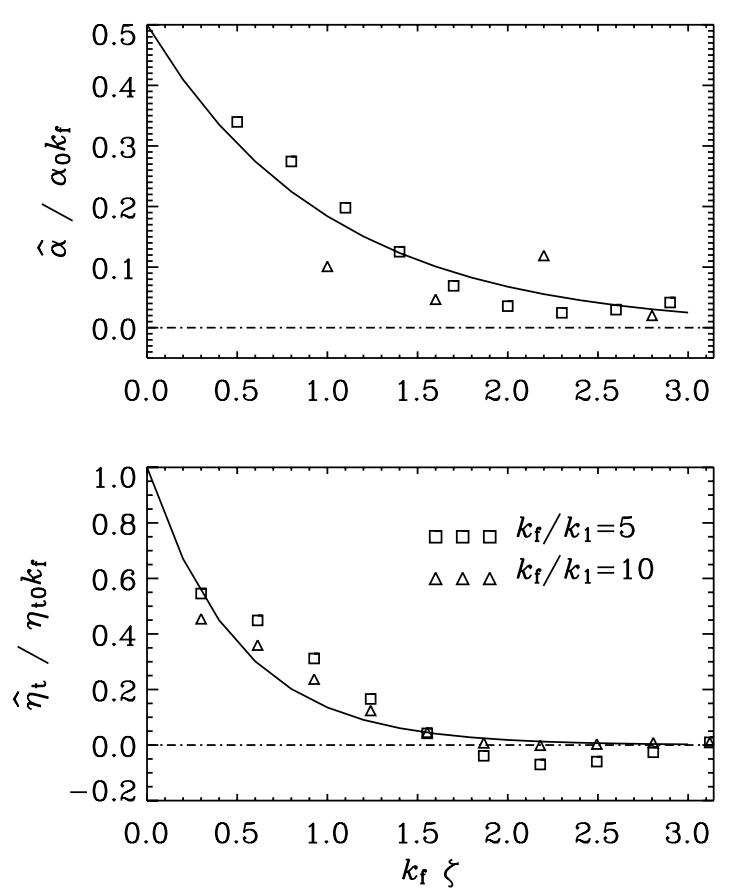

Fig. 5. Normalized integral kernels $\hat{\alpha}$ and $\hat{\eta}_{\mathrm{t}}$ versus $k_{\mathrm{f}} \zeta$ for isotropic turbulence forced at wavenumbers $k_{\mathrm{f}} / k_{1}=5$ with $R_{\mathrm{m}}=10$ (squares) and $k_{\mathrm{f}} / k_{1}=10$ with $R_{\mathrm{m}}=3.5$ (triangles), all with $v / \eta=1$. The solid lines are defined by (36).

it takes a maximum there. Some more details are shown in Fig. 6. With the Lorentzian fits (35) of the results depicted in Fig. 4, we find $\kappa_{0} \approx 0.60$ for $R_{\mathrm{m}}=10$, and $\kappa_{0} \approx 0.45$ for $R_{\mathrm{m}}=3.5$. In the limit of vanishing nonlocal effects it turns out that $\kappa_{0} \approx 0.82$ for $R_{\mathrm{m}}=10$, and $\kappa_{0} \approx 0.59$ for $R_{\mathrm{m}}=3.5$. We have to conclude that dynamos are only possible if the scale of the mean magnetic field clearly exceeds the outer scale of the turbulence. In addition we see again that the nonlocal effect favors smaller $k$, or larger scales of the mean magnetic field.

These findings may become an important issue, especially for nonlinear dynamos or for dynamos with boundaries. Examples of the last kind have been studied, e.g., by Brandenburg \& Sokoloff (2002) and Brandenburg \& Käpylä (2007). In these cases, however, the underlying turbulence is no longer homogeneous, so the kernels $\hat{\alpha}$ and $\hat{\eta}_{\mathrm{t}}$ are no longer invariant under translations; that is, they depend not only on $\zeta$ but also on $z$. The finite widths of the $\hat{\alpha}$ and $\hat{\eta}_{\mathrm{t}}$ kernels may be particularly important if there is also shear, because then there can be a traveling dynamo wave that may also show strong gradients in the nonlinear regime (Stix 1972; Brandenburg et al. 2001).

For another illustration of the significance of a finite width of the kernels $\hat{\alpha}$ and $\hat{\eta}_{\mathrm{t}}$, we consider a one-dimensional nonlinear mean-field model with periodic boundary conditions. We modify here the model of Brandenburg et al. (2001, Sect. 6) with a dynamo number of 10 (corresponding to 5 times supercritical) and $R_{\mathrm{m}}=25$, by introducing the integral kernels (36). Figure 7 shows the components of the mean magnetic field for two different values of $k_{\mathrm{f}} / k_{1}$ and for the conventional case where the kernels are delta-functions. Note that $k_{1}$ corresponds to the largest scale of the magnetic field compatible with the boundary condition. It turns out that the magnetic field profiles are not drastically altered by the nonlocal effect. Low values of $k_{\mathrm{f}} / k_{1}$, however, correspond to smoother profiles. 


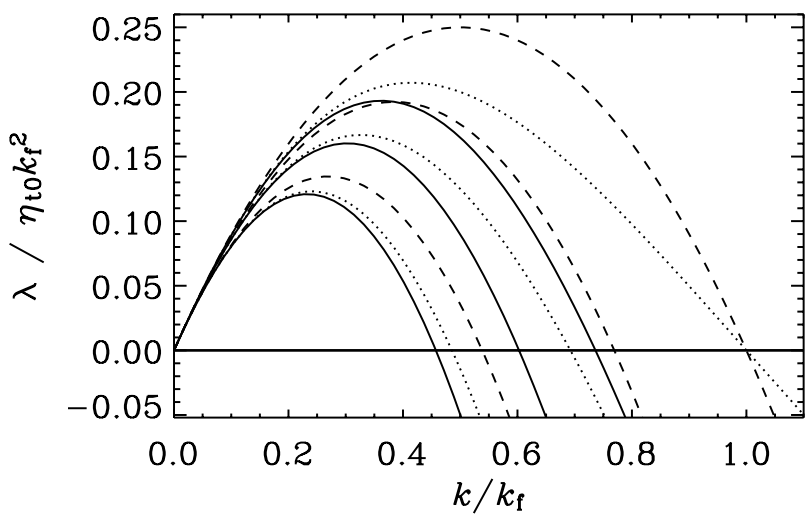

Fig. 6. Normalized growth rate $\lambda(k)$ for isotropic turbulence, calculated according to relation (38) with $\gamma=3$ and with $\tilde{\eta}_{\mathrm{t}} / \eta_{\mathrm{t} 0}$ and $\tilde{\alpha} / \alpha_{0}$ as given in (35), for $R_{\mathrm{m}} \rightarrow \infty$ (upper solid line), as well as $R_{\mathrm{m}}=10$ and 3.5 (next lower solid lines). For comparison, $\lambda$ is also shown for the case in which $\tilde{\eta}_{\mathrm{t}} / \eta_{\mathrm{t} 0}$ coincides with $\tilde{\alpha} / \alpha_{0}$ as given in (35) (dotted lines), and for that of vanishing nonlocal effect in which $\tilde{\eta}_{\mathrm{t}} / \eta_{\mathrm{t} 0}=\tilde{\alpha} / \alpha_{0}=1$ (dashed lines), each for the same three values of $R_{\mathrm{m}}$.
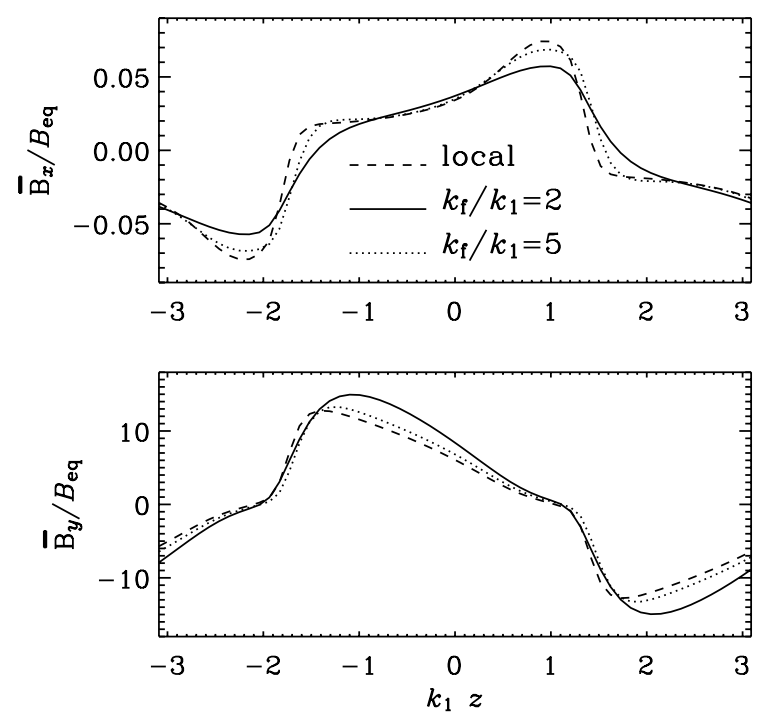

Fig. 7. Mean magnetic field components $\bar{B}_{x}$ and $\bar{B}_{y}$, normalized by the equipartition field strength $B_{\mathrm{eq}}$, in the one-dimensional nonlinear dynamo model characterized in the text, for different values of $k_{\mathrm{f}} / k_{1}$ and for vanishing nonlocal effects.

Let us start again from $\overline{\mathcal{E}}$ in the form (8). We specify there, in view of isotropic turbulence, $\hat{\alpha}$ and $\hat{\eta}_{\mathrm{t}}$ according to (36), and represent $\overline{\boldsymbol{B}}(z-\zeta)$ and $\overline{\boldsymbol{J}}(z-\zeta)$ by Taylor series with respect to $\zeta$. A straightforward evaluation of the integrals provides us then with

$\overline{\mathcal{E}}(z)=\sum_{n \geq 0}\left(\frac{\alpha_{0}}{k_{\mathrm{f}}^{2 n}} \frac{\partial^{2 n} \overline{\boldsymbol{B}}(z)}{\partial z^{2 n}}-\frac{\eta_{\mathrm{t} 0}}{\left(2 k_{\mathrm{f}}\right)^{2 n}} \frac{\partial^{2 n} \overline{\boldsymbol{J}}(z)}{\partial z^{2 n}}\right)$.

This corresponds to relations of the type (1) or (2), generalized simply by taking higher than first-order derivatives of $\overline{\boldsymbol{B}}$ into account.

The terms with derivatives of $\overline{\boldsymbol{J}}$ in (39) can be interpreted in the sense of hyperdiffusion. While all of them have the same signs in real space, the signs of the corresponding terms in Fourier space alternate, which implies that every second term acts in an anti-diffusive manner. Thus, a truncation of the expansion should only be done such that the last remaining term has even $n$, or else anti-diffusion would dominate on small length scales and cause $\overline{\boldsymbol{B}}$ to grow beyond any bound.

There are several investigations in various fields in which hyperdiffusion has been considered. In the purely hydrodynamic context, Rüdiger (1982) derived a hyperviscosity term and showed that this improves the representation of the mean velocity profile in turbulent channel flows. In the context of passive scalar diffusion, Miesch et al. (2000) have determined the hyperdiffusion coefficients for turbulent convection and find that they scale with $n$ like in Eq. (39). We are, however, not aware of earlier studies differentiating between diffusive and anti-diffusive terms.

We investigated the nonlocal cases presented in Fig. 7 using truncations of the expansion (39). However, two problems emerged. Firstly, terms with higher derivatives produce Gibbs phenomena, i.e. wiggles in $\overline{\boldsymbol{B}}$, so the results in Fig. 7 are not reproduced well. Secondly, high-order hyperdiffusion terms tend to give severe constraints on the maximum admissible time step, making this approach less attractive computationally. It appears therefore that a direct evaluation of the convolution terms is most effective.

\section{Conclusions}

The test-field procedure turned out to be a robust method for determining turbulent transport coefficients (see Brandenburg 2005; Sur et al. 2008; Brandenburg et al. 2008). The present paper shows that this also applies to the Fourier transforms of the integral kernels, which occur in the nonlocal connection between mean electromotive force and mean magnetic field, in other words, to the more general scale-dependent version of those transport coefficients. For isotropic turbulence, the kernels $\hat{\alpha}$ and $\hat{\eta}_{\mathrm{t}}$ have a dominant large-scale part and decline monotonously with increasing wavenumbers. This is consistent with earlier findings (cf. Brandenburg \& Sokoloff 2002), where the functional form of the decline however remained rather uncertain. Our present results suggest exponential kernels, corresponding to Lorentzian profiles in wavenumber space. The kernel for the turbulent magnetic diffusivity is about half as wide as for the alpha effect. This result is somewhat unexpected and would be worthwhile confirming before applying it to more refined mean field models. On the other hand, the effects of nonlocality only become really strong when the scale of the magnetic field variations is comparable to or smaller than the outer scale of the turbulence.

One of the areas where future research of nonlocal turbulent transport coefficients is desirable is thermal convection. Here the vertical length scale of the turbulent plumes is often comparable to the vertical extent of the domain. Earlier studies by Miesch et al. (2000) of turbulent thermal convection confirmed that the transport of passive scalars is nonlocal, but it is also more advective than diffusive. It may therefore be important to also allow for nonlocality in time. This would make the expansion of passive scalar perturbations more wave-like, as was shown by Brandenburg et al. (2004) using forced turbulence simulations.

Acknowledgements. We acknowledge the allocation of computing resources provided by the Centers for Scientific Computing in Denmark (DCSC), Finland (CSC), and Sweden (PDC). We thank Matthias Rheinhardt for stimulating discussions. A part of the work reported here was done during stays of K.-H. R. and M.S. at NORDITA. They are grateful for NORDITA's hospitality. 


\section{Appendix A: Justification of Eqs. (5) and (12)}

In view of (5) we start with Eq. (3) for $\overline{\mathcal{E}}$, put $\overline{\mathcal{E}}_{0}=\mathbf{0}$ and assume that $\boldsymbol{K} \circ \overline{\boldsymbol{B}}$ is a purely spatial convolution. Applying then the Fourier transform as defined by $Q(\boldsymbol{x})=\int \tilde{Q}(\boldsymbol{k}) \exp (\mathrm{i} \boldsymbol{k}$. $\boldsymbol{x}) \mathrm{d}^{3}(k / 2 \pi)$, we obtain

$\tilde{\overline{\mathcal{E}}}_{i}(\boldsymbol{k})=\tilde{K}_{i j}(\boldsymbol{k}) \tilde{\bar{B}}_{j}(\boldsymbol{k})$.

Since $\overline{\mathcal{E}}$ and $\overline{\boldsymbol{B}}$ have to be real, we conclude that $\tilde{K}_{i j}^{*}(\boldsymbol{k})=$ $\tilde{K}_{i j}(-\boldsymbol{k})$. Furthermore, the assumption of isotropic turbulence requires that the components of $\tilde{K}_{i j}$ are invariant under arbitrary simultaneous rotations of $\tilde{\boldsymbol{u}}$ and $\boldsymbol{k}$. We have therefore

$\tilde{K}_{i j}=\tilde{\alpha}(k) \delta_{i j}+\tilde{\alpha}^{\prime}(k) k_{i} k_{j}+\mathrm{i} \tilde{\eta}_{\mathrm{t}}(k) \epsilon_{i j k} k_{k}$

with $\tilde{\alpha}, \tilde{\alpha}^{\prime}$, and $\tilde{\eta}_{\mathrm{t}}$ being real functions of $k=|\boldsymbol{k}|$. Considering further that $\boldsymbol{k} \cdot \tilde{\overline{\boldsymbol{B}}}=0$ and $\mathrm{i} \boldsymbol{k} \times \tilde{\overline{\boldsymbol{B}}}=\tilde{\overline{\boldsymbol{J}}}$, we find

$\tilde{\overline{\mathcal{E}}}(\boldsymbol{k})=\tilde{\alpha}(k) \tilde{\overline{\boldsymbol{B}}}(\boldsymbol{k})-\tilde{\eta}_{\mathrm{t}}(k) \tilde{\overline{\boldsymbol{J}}}(\boldsymbol{k})$.

Transforming this in physical space we immediately obtain (5).

In view of (12) we start again from Eq. (3) and put $\overline{\boldsymbol{\varepsilon}}_{0}=$ $\mathbf{0}$, but we have to consider $\boldsymbol{K} \circ \overline{\boldsymbol{B}}$ now as a convolution only with respect to $z$. Applying a Fourier transformation defined by $Q(z)=\int \tilde{Q}(k) \exp (\mathrm{i} k z) \mathrm{d}(k / 2 \pi)$, we obtain a relation analogous to (A.1),

$\tilde{\overline{\mathcal{E}}}_{i}(k)=\tilde{K}_{i j}(k) \tilde{\bar{B}}_{j}(k)$,

and may now conclude that $\tilde{K}_{i j}^{*}(k)=\tilde{K}_{i j}(-k)$. We thus arrive at

$\tilde{K}_{i j}=\tilde{\alpha}_{i j}(k)+\mathrm{i} k \tilde{\eta}_{i j}^{\prime}(k)$

with real tensors $\tilde{\alpha}_{i j}$ and $\tilde{\eta}_{i j}^{\prime}$, which are even in $k$. Combining (A.4) and (A.5), considering that $\mathrm{i} k \tilde{\bar{B}}_{1}=\tilde{\bar{J}}_{2}, \mathrm{i} k \tilde{\bar{B}}_{2}=$ $-\tilde{\bar{J}}_{1}$ and $\mathrm{i} k \tilde{\bar{B}}_{3}=0$, and putting $\mathrm{i} k \tilde{\eta}_{i j}^{\prime}=\tilde{\eta}_{i j} \tilde{\bar{J}}_{j}$ with a proper tensor $\tilde{\eta}_{i j}$, we may confirm first (13) and so also (12).

\section{Appendix B: Mean-field results for the Roberts flow}

A mean-field theory of the Roberts dynamo, developed in view of the Karlsruhe dynamo experiment, has been presented, e.g., in papers by Rädler et al. (2002a,b), in the following referred to as R02a and R02b. There a fluid flow like the one given by (26) is considered but without any coupling of its magnitudes in the $x y$-plane and in the $z$-direction. The mean fields are defined by averaging over finite areas in the $x y$-plane so that they may still depend on $x$ and $y$ in addition to $z$. As shown in those papers, when contributions with higher than first-order derivatives of $\overline{\boldsymbol{B}}$ are ignored, then $\overline{\mathcal{E}}$ has the form

$$
\begin{aligned}
\overline{\mathcal{E}}= & -\alpha_{\perp}[\overline{\boldsymbol{B}}-(\hat{z} \cdot \overline{\boldsymbol{B}}) \hat{z}]-\beta_{\perp} \boldsymbol{\nabla} \times \overline{\boldsymbol{B}}-\left(\beta_{\|}-\beta_{\perp}\right)[\hat{z} \cdot(\boldsymbol{\nabla} \times \overline{\boldsymbol{B}})] \hat{z} \\
& -\beta_{3} \hat{z} \times[\boldsymbol{\nabla}(\hat{z} \overline{\boldsymbol{B}})+(\hat{z} \cdot \boldsymbol{\nabla}) \overline{\boldsymbol{B}}]
\end{aligned}
$$

with constant coefficients $\alpha_{\perp}, \beta_{\perp}, \beta_{\|}$, and $\beta_{3}$ (see (9) of R02a or (9) of R02b). Reducing this to the case considered above, in which $\bar{B}$ no longer depends on $x$ and $y$, we find

$\overline{\mathcal{E}}=\alpha[\overline{\boldsymbol{B}}-(\hat{z} \cdot \overline{\boldsymbol{B}}) \hat{z}]-\eta_{\mathrm{t}} \boldsymbol{\nabla} \times \overline{\boldsymbol{B}}$,

where $\boldsymbol{\nabla} \times \overline{\boldsymbol{B}}=\hat{z} \times \partial \overline{\boldsymbol{B}} / \partial z$, and

$\alpha=-\alpha_{\perp}, \quad \eta_{\mathrm{t}}=\beta_{\perp}+\beta_{3}$.
Results for $\alpha_{\perp}, \beta_{\perp}, \beta_{\|}$, and $\beta_{3}$ obtained under SOCA are given in (19) and (38) of R02a, as well as in (19) and (49) of R02b. When fitting them with $u_{\perp}=(2 / \pi) u_{0}, u_{\|}=\sqrt{2}(2 / \pi)^{2} u_{0}$, $\pi / a=k_{0}, \sqrt{2} \pi / a=k_{\mathrm{f}}, R_{\mathrm{m} \perp}=\sqrt{2} R_{\mathrm{m}}$, and $R_{\mathrm{m} \|}=(8 / \pi) R_{\mathrm{m}}$ to the assumptions and notations used above, we find just (30). Likewise (20) of R02a and (20) of R02b lead to (31).

\section{Appendix C: $\tilde{\alpha}$ and $\tilde{\eta}_{\mathrm{t}}$ under SOCA for Roberts flow}

Let us start with the relation (B.2) and subject it to a Fourier transformation with respect to $z$ so that

$\tilde{\overline{\mathcal{E}}}=\overline{\boldsymbol{u} \times \tilde{\boldsymbol{b}}}=\tilde{\alpha}[\tilde{\overline{\boldsymbol{B}}}-(\hat{z} \cdot \tilde{\overline{\boldsymbol{B}}}) \hat{z}]-\mathrm{i} k \tilde{\eta}_{\mathrm{t}} \hat{z} \times \tilde{\overline{\boldsymbol{B}}}$

From the induction equation, we have

$\eta\left(\boldsymbol{\nabla}^{2}-k^{2}\right) \tilde{\boldsymbol{b}}=-(\boldsymbol{\nabla}+\mathrm{i} k \hat{z}) \times(\boldsymbol{u} \times \tilde{\overline{\boldsymbol{B}}}), \quad k \tilde{b}_{z}=0$.

The solution $\tilde{\boldsymbol{b}}$ reads

$$
\begin{aligned}
\tilde{\boldsymbol{b}}= & -\frac{1}{\eta\left(k^{2}+k_{\mathrm{f}}^{2}\right)}\left\{\hat{z} \times \nabla(\tilde{\overline{\boldsymbol{B}}} \cdot \nabla \psi)-k_{\mathrm{f}}(\tilde{\overline{\boldsymbol{B}}} \cdot \nabla \psi) \hat{z}\right. \\
& \left.+\mathrm{i} k\left[\hat{z} \times \nabla \psi(\hat{z} \cdot \tilde{\bar{B}})+k_{\mathrm{f}} \psi(\tilde{\overline{\boldsymbol{B}}}-(\hat{z} \cdot \tilde{\bar{B}}) \hat{z})\right]\right\},
\end{aligned}
$$

where $\boldsymbol{u}$ is expressed by $\psi$ according to (24). Calculate now $\tilde{\overline{\mathcal{E}}}_{x}$ or $\tilde{\overline{\mathcal{E}}}_{y}$ utilizing $\overline{\psi^{2}}=\frac{1}{4}\left(u_{0} / k_{0}\right)^{2}, \overline{(\partial \psi / \partial x)^{2}}=-\overline{\psi \partial^{2} \psi / \partial x^{2}}=\frac{1}{4} u_{0}^{2}$ and $\overline{\partial \psi / \partial x \partial \psi / \partial y}=\overline{\psi \partial^{2} \psi / \partial x \partial y}=0$. When comparing the result with (C.1) we immediately find (32). Using then relations of the type (14), we also find (33).

\section{References}

Blackman, E. G., \& Field, G. B. 2002, PRL, 89, 265007 Brandenburg, A. 2005, AN, 326, 787 Brandenburg, A., \& Sokoloff, D. 2002, GApFD, 96, 319

Brandenburg, A., \& Subramanian, K. 2005a, PhR, 417, 1

Brandenburg, A., \& Subramanian, K. 2005b, A\&A, 439, 835

Brandenburg, A., \& Käpylä, P. J. 2007, New J. Phys., 9, 305

Brandenburg, A., Bigazzi, A., \& Subramanian, K. 2001, MNRAS, 325, 685

Brandenburg, A., Käpylä, P., \& Mohammed, A. 2004, PhFl, 16, 1020

Brandenburg, A., Rädler, K.-H., Rheinhardt, M., \& Käpylä, P. J. 2008, ApJ, 676, 740

Dobler, W., Haugen, N. E. L., Yousef, T. A., \& Brandenburg, A. 2003, PRE, 68, 026304

Krause, F., \& Rädler, K.-H. 1980, Mean-Field Magnetohydrodynamics and Dynamo Theory (Oxford: Pergamon Press)

Miesch, M. S., Brandenburg, A., \& Zweibel, E. G. 2000, PRE, 61, 457

Moffatt, H. K. 1978, Magnetic field generation in electrically conducting fluids (Cambridge: Cambridge University Press)

Rädler, K.-H. 2000, in From the Sun to the Great Attractor, ed. D. Page, \& J. G. Hirsch, Lect. Notes Phys., 556, 101

Rädler, K.-H., \& Brandenburg, A. 2003, PRE, 67, 026401

Rädler, K.-H., \& Rheinhardt, M. 2007, GApFD, 101, 11

Rädler, K.-H., Rheinhardt, M., Apstein, E., \& Fuchs, H. 2002a, Magnetohydrodynamics, 38, 41 (R02a)

Rädler, K.-H., Rheinhardt, M., Apstein, E., \& Fuchs, H. 2002b, Nonl. Processes Geophys., 38, 171 (R02b)

Roberts, G. O. 1972, Phil. Trans. Roy. Soc., A 271, 411

Rüdiger, G. 1982, Zeitschr. Angewandt. Math. Mech., 6, 95

Schrinner, M., Rädler, K.-H., Schmitt, D., Rheinhardt, M., \& Christensen, U. 2005, AN, 326, 245

Schrinner, M., Rädler, K.-H., Schmitt, D., Rheinhardt, M., \& Christensen, U. R. 2007, GApFD, 101, 81

Stix, M. 1972, A\&A, 20, 9

Sur, S., Brandenburg, A., \& Subramanian, K. 2008, MNRAS, 385, L15 